\title{
Técnicas para la visualización de dominios científicos y tecnológicos
}

\author{
Maidelyn Díaz Pérez* \\ Félix de Moya Anegón ${ }^{\star *}$ \\ Humberto Andrés Carrillo-Calvet***
}

Artículo recibido:

22 de enero de 2016

Artículo aceptado:

15 de abril de 2016

\section{RESUMEN}

Esta investigación consiste en una revisión de aspectos considerados como los más importantes en el desarrollo y evolución de la visualización de información en la representación de dominios científicos. Su objetivo es distinguir las técnicas más apropiadas para visualizar dominios, pero, en este caso, no de información científica (artículos), sino de información tecnológica (patentes). Esta investigación compila teorías, tendencias y contribuciones relevantes sobre visualización de información, en particular análisis referentes a la visualización

* Universidad de Pinar del Río, Cuba. maidelyn@upr.edu.cu.

** Consejo Superior de Investigaciones Científicas (CSIC), España.felix.moya@scimago.es.

*** Facultad de Ciencias, UNAM, México. carr@unam.mx.

INVESTIGACIÓN BIBLIOTECOLÓGICA, Número Especial de Bibliometría, 2017, México, ISSN: 2448-8321, pp. 17-42 
bibliométrica y algunas apreciaciones significativas sobre las principales técnicas de visualización utilizadas para el análisis de dominios científicos, principalmente tecnológicos. Esto permite llegar a conclusiones que asisten la selección de las técnicas más adecuadas para visualizar la producción tecnológica de diferentes dominios, teniendo como valor agregado un inventario de las principales tendencias científicas y tecnológicas más relevantes en la visualización de dominios en el campo de la ciencia de la información.

Palabras clave: Visualización de información; Visualización bibliométrica; Mapas; Técnicas de visualización; Análisis de redes sociales; Visualización de dominios tecnológicos.

Techniques to visualize of technology and scientific domains

Maidelyn Diaz Pérez, Félix de Moya Anegón and Humberto Andrés Carrillo-Calvet

\section{Abstract}

This research consists of a review of issues considered most important in the development and evolution of the information display in the representation of scientific domains. It aims to distinguish the most appropriate techniques to display domains, but in this case, no Scientific Information (articles) but of Information Technology (patents). This research compiled theories, trends and relevant contributions on information visualization, especially concerning bibliometric analysis and display some significant insights into the main visualization techniques used for analysis, mainly technologi$\mathrm{cal}$ and scientific domains. This allows conclusions to attend the selection of the most appropriate techniques to display the technological production of different domains, taking as an added value an inventory of the main display most relevant domains in the field of infor- 
Keywords: Information visualization; Bibliometric visualization; Map visualization; Visualization techniques; Social network analysis; Technology domains visualization.

\section{INTRODUCCIÓN}

Si dedicamos un tiempo a pensar en algunos de los principales aportes que han caracterizado el desarrollo de la humanidad en sus diferentes épocas y periodos, resulta fácil recordar la aparición de la imprenta, la fotografía, la televisión, el telescopio, etc., contribuciones cuya esencia revela información en diferentes formatos e imágenes. Incluso la aparición del lenguaje escrito, tras la elaboración, durante siglos, de diversos trazos y signos, no es más que uno de los primeros intentos por tratar de asociar imágenes a sonidos con significado, o sea, visualizar el lenguaje oral. Una visualización sería una figura, foto, diagrama tridimensional, etc.; lo esencial de una visualización o imagen es que sea posible interactuar con ésta; interacción que permite gestionar las visualizaciones de la misma forma que se gestionan otros objetos y hechos de la realidad. El campo de la visualización es tan amplio que permite hacer visible al ojo humano lo casi imperceptible a la visión (estructura molecular), además de hacer visible, mediante la reducción de la dimensión espacial, algo tan grande como nuestro planeta; sin olvidar su gran potencial para revelar entidades no físicas que no adquieren una forma visual. Este último es el objeto de la visualización de información (VI), ya que permite volver apreciable al ojo humano sucesos y comportamientos no perceptibles en sí, y esta técnica se aplica tanto a los documentos científicos como a los tecnológicos. Esta investigación tiene especial interés en la literatura tecnológica, y dentro de ésta, en los documentos de patentes.

Las patentes son la fuente de información que por excelencia utilizan los estudios de innovación tecnológica: una de las fuentes de datos más válidas para realizar análisis métricos. Sus potencialidades le han permitido ser considerada, incluso, una unidad de medida confiable para analizar la productividad científico-tecnológica de un país, región, industria, tecnología, etcétera.

Los indicadores de tecnología basados en patentes se han utilizado desde hace varias décadas, sin embargo, ha primado en muchos una concepción teoricista, en la que la ciencia consiste en supuestos y formulaciones teóricas, mientras que la tecnología se ocupa de patentes, sin relación alguna con artefactos materiales, conjeturando entonces que la tecnología es sólo técnica y ciencia aplicada; concepción que en la actualidad no se corresponde con los desarrollos alcanzados en los ámbitos científicos, tecnológicos 
y de innovación, demostrados en muchos estudios métricos con información de patentes.

Hoy, los márgenes entre ciencia y tecnología cada vez se tornan más difusos y difíciles de observar, de ahí la complejidad para definir y elaborar indicadores que reflejen, fielmente, la realidad innovadora y tecnológica de cualquier dominio. En la revisión documental realizada para esta investigación, se constató que, tal y como existen metodologías para el análisis del sistema de ciencia, basadas en indicadores de producción científica (artículos), hay otras que sirven para medir y evaluar el desarrollo tecnológico (Meyer et al., 2004; Meyer y Bhattacharya; 2006; Meyer, 2006), utilizando diferentes técnicas para la representación y visualización de la información. Instituyéndose para ello las patentes como la fuente de información más confiable y relevante que permite medir la producción tecnológica de un dominio. Los estudios más significativos sobre la importancia del uso métrico de la información de patentes comienzan a publicarse en la década del sesenta. Y años más tarde ya se empieza a hablar de la patentometría como un equivalente al análisis bibliométrico de patentes (Narin, 1994), convirtiéndose, desde entonces, en uno de los análisis de mayor utilidad para las industrias, porque permite un mayor acercamiento a la realidad tecnológica (Narin et al., 1995; 1997); así como identificar nichos tecnológicos de firmas, proyectos de investigación, centros, etc. (Stuart y Podolny, 1996); identificar redes de innovación en el desarrollo de tecnologías (Griliches, 1990; Archibugi, 1992) y posibles relaciones entre sí (ciencia-tecnología) (Brooks, 1994). Además de utilizar las patentes para evaluar las investigaciones tecnológicas y la evolución de las innovaciones introducidas en el mercado internacional (Trajtenberg; 1990). Pero, muy a pesar de todas sus fortalezas, aún no existen suficientes estudios sobre el uso de técnicas para la representación y visualización de dominios tecnológicos, en aras de fortalecer su aplicación en estudios métricos de monitoreo y vigilancia tecnológica, así como para otros fines investigativos. El estado de la cuestión constató que existe poca bibliografía científica que compile las técnicas existentes para estos fines.

Esta investigación reúne teorías, tendencias y contribuciones relevantes sobre visualización de información, en particular análisis referentes a la visualización bibliométrica y algunas apreciaciones significativas sobre las principales técnicas de visualización utilizadas para el análisis de dominios científicos, principalmente tecnológicos. Esto permite llegar a conclusiones que asisten la selección de las técnicas más adecuadas para representar la producción tecnológica de diferentes dominios, teniendo como valor agregado un inventario de las principales tendencias científicas y tecnológicas en la visualización de dominios en el campo de la ciencia de la información. 


\section{Métodos}

Esta investigación utilizó diferentes métodos teóricos para analizar, con el rigor científico requerido, el tema objeto de estudio:

- Método histórico y lógico: proporcionó un correcto análisis de los referentes históricos y teóricos que respaldan las tendencias actuales sobre las técnicas para la visualización de dominios científicos y tecnológicos.

- Método análisis y síntesis: facilitó todo el proceso de identificación, análisis y sistematización de la bibliografía sobre el tema a nivel internacional, facilitando la comprensión de su evolución en el tiempo. La aplicación de este método permitió nivelar las principales tendencias relacionadas con las técnicas para la visualización de dominios científicos y tecnológicos, logrando sintetizar los aspectos más novedosos vinculados con el objetivo de esta investigación.

- Método inducción-deducción: facilitó la disertación del tema desde la articulación filosófica de lo general, lo particular y lo singular, incluyendo diferentes valoraciones filosóficas que permiten una aproximación más práctica a la realidad actual. Este método ayudó al análisis pragmático del uso de las diferentes técnicas para la visualización de dominios científicos y tecnológicos, propiciando un entendimiento objetivo de cual seleccionar para analizar cada tipo de dominio.

\section{VISUALIZACIÓN DE LA INFORMACIÓN}

Una de las definiciones pioneras encontradas sobre visualización la aporta Jacques Bertin, cartógrafo y teórico francés, conocido por su libro Sémiologie Graphique (Semiología de los gráficos) de 1967. Este libro, elaborado a partir de su experiencia como cartógrafo y geógrafo, representa la primera y más amplia intención de proporcionar una base teórica a la VI. Para Bertin, la finalidad de la VI era optimizar el uso de la percepción humana y la habilidad del pensamiento para tratar con fenómenos que por sí solos no pueden ser representados de forma visual en un espacio bidimensional. Antes que Bertin publicara el libro sobre la teoría de gráficos, existen otros antecedentes, como los de Bradford y Bush. En 1945, cuando Vannevar Bush (1945) publicó el artículo "As we may think" en la revista Athlantic Monthtly, ya estaba pensando en algo similar, al mencionar un sistema capaz de unir la información por enlaces asociativos, sistema al cual denominó Memex ("Memory Extended"), idea desarrollada en 1963 por Ivan Sutherland, quien desarrolló 
el Sketchpad, primer entorno gráfico de la historia inspirado en el Memex de Bush, y que permitía manipular objetos visibles a través de un lápiz óptico, cambiar su tamaño y moverlos, entre otras cosas.

Bradford (1948), al referirse a la idea de que la ciencia podía ser visualizada o mapeada, la veía como un gráfico del universo en el que los discursos aparecen distribuidos en una esfera, en una confusión promiscua, mutuamente relacionados y separados de las cosas que vemos o que pensamos. Estas nacientes aproximaciones al tema revelan que desde hace varias décadas existe interés en buscar nuevas formas de representación de la información. Otro de los primeros en articular esta necesidad fue Doyle (1961), quien avizoró la importancia de los ordenadores, para producir mapas similares a los que genera el cerebro, indicando cómo podían ser proyectados en espacios multidimensionales, e incluso da su opinión sobre el objetivo de dichos mapas y cómo construirlos. Unos años más tarde, Garfield (1963) hizo público en la American Documentation su gran interés por la construcción de mapas históricos mediante el uso de citas. Idea que desarrolló junto a Irv Sher en 1964, al realizar un mapa histórico en el que se mostraba el desarrollo del adn desde Mendel hasta Niremberg. Desde ese año, el autor trabajó en conjunto con otros autores, generando mapas históricos topológicos de la ciencia, a partir de citas de la producción científica y utilizando como variable el emparejamiento bibliográfico "bibliographic coupling" (Garfield et al., 1964). Un aporte significativo en los sesenta que propició nuevos desarrollos en el campo de la representación de grandes dominios de información científica lo tributa De Solla (1965), quien demostró que los patrones de citas utilizados por los autores de artículos científicos definían los frentes de investigación, y que éstos se aprovecharían para delinear una topología que reflejase la estructura de la producción científica. Durante los años sesenta, también emergió la lingüística textual, dentro de las aplicaciones lingüísticas en la documentación. Se emprendieron investigaciones (Moreiro, 1998) sobre los mecanismos lógico-semánticos inherentes a todo texto, donde las operaciones de análisis intentan alcanzar sistemas simbólicos de representación. En concordancia con este desarrollo, en 1965, Ted Nelson creó el hipertexto, con la finalidad de organizar el universo de documentos a partir de una estructura lineal basada en relaciones intertextuales.

La década del setenta tuvo un desarrollo considerable en términos de visualizar datos desde imágenes y gráficos, gracias a la expansión de las computadoras de bajo costo, aunque también influyó el auge de los sistemas de información geográfica (gis), con base en la cartografía tradicional. Contribución significativa en el uso de metáforas visuales, con un enfoque geográfico (Börner et al., 2003). Esta investigación coincide con el avance más 
notable del decenio, las aportaciones de Small (1973) y Marshakova (1973), sobre los diseños de mapas o gráficos de un dominio. Quienes propusieron de forma independiente la cocitación de documentos, como variable de estudio en los análisis de citas de la producción científica. Y utilizan dicha variable de estudio y el Science Citation Index (SCI) como fuente de información, un año más tarde, elaboraron mapas de la ciencia, en los que se muestran todas las especialidades de las ciencias naturales (Small y Griffith, 1974; Griffith et al., 1974), ya que la metodología empleada por los autores identificaba perfectamente los grupos de documentos que tenían intereses intelectuales comunes. Se demuestra entonces que la ciencia es una red de especialidades interconectadas entre sí, y que se representan si utilizan como base el análisis cuantitativo de la producción escrita.

Aaronson (1975) aplica las teorías anteriores y radiografía la biomedicina de los años 1972-1973, e identifica en el mapa de 1973 el llamado superclúster porque muestra la convergencia con otras especialidades. Los mapas de Aaronson no sólo desplegaron la representación gráfica de un domino y reflejaron su evolución en el tiempo, sino que además manifestaron cómo las distintas disciplinas interactuaban entre sí, ofreciendo incluso información sobre los documentos que lo integraban (denominación, número de documentos que lo componen, grado de conexión entre documentos representado por líneas acompañadas del valor de cocitación que los conectan, etc.). De esta forma, se identifican los clústers más importantes, en función del mayor número de relaciones con el resto. Es indiscutible que los mapas de Aaronson son una referencia reveladora que trazó el camino en la evolución y estudio de la representación y visualización de dominios científicos hasta el presente.

En los ochenta, se publicó, tras su anuncio seis años ante, por parte de Garfield, el Atlas de la Ciencia, elaborado por el Institute for Science Information (ISI). En 1981, aparecieron los dos primeros volúmenes del Atlas: Bioquímica y Biología molecular (Garfield, 1981), y después se publicaron otros volúmenes del Atlas de la Ciencia (Garfield, 1984; 1988): Biotecnología y genética molecular; Bioquímica, inmunología y biología animal y vegetal. Estos mapas, a pesar de haber sido elaborados por el ISI, las técnicas que utilizan para su generación no difieren mucho de las utilizadas por Small y Griffith (1974). Usan la generación de clústers a partir de los datos obtenidos por la cocitación de documentos (variable de relación) en una disciplina concreta, la novedad y aporte principal lo constituyó el uso de un nuevo posicionamiento espacial de los clusters, mediante técnicas de escalamiento multidimensional (MDS).

Esta investigación considera que esos primeros resultados mencionados hasta aquí constituyen los pilares fundamentales de la disciplina VI, 
convirtiéndose en verdaderos paradigmas dentro del desarrollo de la representación de dominios científicos. Desde estas premisas, es que surgen trabajos posteriores que definen metodologías de trabajo y técnicas de aplicación.

En la segunda mitad de los ochenta, se comienza a apreciar algo que no debe ignorarse en este estudio cronológico evolutivo sobre el tema, por primera vez existía, paralelamente, junto a la academia, un interés gubernamental por la visualización de información. Interés manifestado en un acto científico patrocinado por la National Science Fundation (NSF) de Estados Unidos, quienes presentaron un informe sobre visualización. Aquí se discutieron muchas inquietudes sobre "el dilema de la información sin interpretación", pues en los años ochenta había tantas fuentes de datos (satélites, radares, escáneres, etc.) como dificultades para procesar e interpretar la información compleja que suministraban; en esencia, un verdadero problema, mas no el único.

Fue interés de este informe discutir también cómo mejorar la comunicación de los resultados científicos, y cómo conseguir una mayor interacción entre los investigadores y el análisis informático de los datos. Se convertía en una prioridad el desarrollo de hardware, software y herramientas visuales para gráficos y técnicas de procesamiento de imágenes. Y dentro de toda explosión informacional, en octubre de 1986 se revela la importancia de la computadora como herramienta intermediaria en el proceso de la asimilación rápida de la información (Robertson et al., 1999).

Un informe de la NSF maneja el concepto de visualización en el dominio científico, definiéndola como un método para transformar lo simbólico en geométrico, y como una herramienta para interpretar datos de imágenes introducidos en una computadora, generar imágenes de conjuntos de datos complejos multidimensionales y estudiar los mecanismos en los humanos y computadoras que les permitan, de manera conjunta, percibir, usar y comunicar la información visual, considerándose entonces la visualización científica como una disciplina especial (McCormick et al., 1987). Junto a la NSF, en los años ochenta, los estudios en visualización de información fueron promovidos por el Human-Computer Interaction Lab (HCIL) de la Universidad de Maryland, creado en 1983 por Ben Shneiderman y el Centro de Investigación de la Xerox de Palo Alto (Xerox PARC), quienes tenían interés en aplicar las experiencias de la visualización científica a nuevos campos que permitieran manipular datos más complejos, manejar mayores volúmenes de información y trabajar con diferentes tipologías documentales.

Durante estos años, se generan trascendentales apreciaciones que fortalecieron el campo, tanto de la visualización informática, como de la visualización de información. Los investigadores empiezan a comprender que 
necesitan algo más que números; y los estudiosos del tema afirman que la necesidad técnica del hoy y el imperativo cognitivo del mañana es el uso de la imagen. La habilidad de los investigadores para visualizar cálculos informáticos complejos, así como simulaciones, es esencial para asegurar la integridad de los análisis, al mismo tiempo que provocan la aparición de nuevos conocimientos que serían transmitidos a otros (McCormick et al., 1987: 18).

El estado de la cuestión refiere que el término de visualización de información se utilizó por primera vez por Robertson et al. (1999: 28) en 1989, reseñando una propuesta de arquitectura cognitiva para interfaces de usuario interactivas. En ese momento, los autores la consideraron análoga a la visualización científica, y en ella proponían el uso de objetos animados bidimensionales (2D) y tridimensionales (3D) para representar la información y sus relaciones estructurales. En las postrimerías de los años ochenta, se marcó el inició de una búsqueda por encontrar nuevas definiciones que expresaran los verdaderos propósitos científicos de la VI y el porqué la visualización puede utilizarse como elemento de análisis e interpretación de datos e informaciones. Para dar respuesta a esta interrogante, surgieron las propuestas de Robertson et al. (1999: 17), quienes describieron el propósito de la visualización como el uso de la visión de pensar, donde pensar es entendido como un elemento central del conocimiento, de donde se deriva el principio de adquisición de conocimiento mediante imágenes. Estos autores consideran a la VI como el uso de soportes computacionales interactivos que permiten realizar representaciones visuales de los datos para ampliar la cognición; la cognición se considera el equivalente de conocimientos, saberes, entendimientos, etc. En la VI, la interacción de los individuos con las imágenes o visualizaciones para la generación de nuevas ideas y conocimientos es primordial. Argumentos que avalan, junto a otros criterios, la idea de que la visualización tiene la habilidad especial de activar controladamente los refinados mecanismos de la percepción visual humana.

En la misma década, otro grupo de autores definieron experimentalmente que las representaciones visuales eran superiores a las representaciones verbal-secuenciales en diversas tareas (Larkin y Simon, 1987; Glenberg y Langston, 1992). Otros la limitaron a un proceso asistido por la computadora, en el cual se busca revelar señales de un fenómeno abstracto al transformar datos en formas visuales.

Gershon y Pages (2001) consideran la VI como un "proceso que transforma datos, información, y conocimiento en una forma que permite al sistema visual humano percibir la información de forma integrada". Más que un simple resultado o acción única, es todo un proceso que permite al analista, mediante la visión óptica, hacer lecturas apropiadas de la información que se visualiza. Mientras existen otros autores (Eick, 2001) que sólo la conciben 
y enmarcan en la creación de interfaces visuales que permiten auxiliar a los usuarios a entender y navegar a través de espacios complejos de la información.

Dürsteler, uno de los investigadores que mejor ha tratado este tema, conceptualiza la VI como "el uso de soportes computacionales interactivos que permiten realizar representaciones visuales de los datos para ampliar la cognición”. Es como la formación de la imagen mental de un concepto abstracto. La imagen —y por tanto la visualización- es una construcción mental que va más allá de la percepción sensorial y que, como tal, se acerca al conocimiento como la aprehensión intelectual de las cosas.

Börner et al. (2003: 8), al hacer una profunda revisión bibliográfica sobre el tema, declararon la VI como una posibilidad de diseñar la apariencia visual de los objetos de datos y sus relaciones. En el mismo año, otros autores (Hearst, 2003) reconocieron nuevas facilidades que posee para la comparación, reconocimiento de patrones, detección de cambios y otras habilidades cognitivas para hacer uso del sistema visual, a partir de representaciones espaciales o gráficas de la información. En igual sentido, hay investigadores (Zhu y Chen, 2005) que no sólo la definen para representar de forma visual espacios y estructuras de información que faciliten una rápida asimilación y comprensión, sino que resaltan su posibilidad de identificar y extraer patrones a partir de una gran cantidad de información que incrementa su valor.

Joan Costa (1998) expone criterios muy interesantes y valederos al considerar que visualizar no es el resultado implícito del acto de ver, no es un producto espontáneo del individuo que recibe la información ya visualizada. Aquél requiere también la comprensión del individuo que observa e interpreta la visualización, 4 quien debe ser capaz de inferir información que yace oculta. El dominio de los lenguajes gráficos para visualizar estos "efectos invisibles” configura una nueva ciencia de la comunicación visual, la esquemática, a la que Costa ha definido como el "tercer lenguaje" después de la imagen y el signo. Esta última muy estudiada por Herrero (2000) en el área de la documentación.

Polanco y Zarti (2008) comparten otro concepto de visualización: la visualización científica y la de información. Y a la par plantean que la naturaleza de estas distinciones es empírica, fundamentada por las diferencias de los datos de entrada y salida (datos visualizados). Al transferir estas perspectivas hacia la bibliometría, identifican la visualización bibliométrica como la representación de indicadores bibliométricos y sus resultados, a

4 El dominio de los lenguajes gráficos para visualizar estos "efectos invisibles" configuran una nueva ciencia de la comunicación visual, la esquemática, a la que Costa (1998) ha definido como el "tercer lenguaje" después de la imagen y el signo. 
los que se han aplicado algunas de las técnicas de visualización de información existentes para mejorar la interpretación del dominio. A partir de esta aplicación, se representaría mejor la imagen visual de las relaciones entre documentos, detectar los autores más importantes de una determinada disciplina, analizar las estructuras de un área de conocimiento $\mathrm{X}$, así como su evolución en el tiempo, ya que permite identificar relaciones entre una gran cantidad de variables.

La VI, por sus características propias, tiene, de hecho, muchas funciones, pero las más importantes para esta investigación son su posibilidad de descubrir los patrones que subyacen a los datos, describir el conocimiento existente y a su vez inferir cualquier nuevo conocimiento, así como ser capaz de transmitir ese mensaje y que sea comprensible por el ojo humano.

En este estudio se constata, con la revisión de toda la información antecedente, que no es algo nuevo la necesidad de representar la información científica de una forma más inteligible para la mente humana. Hacer visible para nuestra mente "lo que no es visible para nuestros ojos [...] y crear una imagen mental de algo, aquello que no se ve a simple vista, una abstracción, etc. son definiciones de la palabra visualización que ponen de manifiesto la necesidad intrínseca de representar la información de una forma distinta a la tradicional (De Moya et al., 2004).

Otro de los hallazgos de esta investigación es comprender el uso dado a este término, en función de su aplicación y resultados, refiriéndose los autores indistintamente a la VI como disciplina, proceso o instrumento.

La representación gráfica y la VI no son actividades tan nuevas, desde el siglo xx se trabaja en éstas por parte de investigadores procedentes de diversas disciplinas científicas. Aunque la VI sea una práctica antigua, se trata de una disciplina científica reciente, considerada un campo de estudio emergente de la ciencia que avanza vertiginosamente en términos de investigaciones académicas y aplicaciones prácticas. Su basamento es interdisciplinar (Torres, 2007), marcado por investigaciones y enfoques aportados por otras disciplinas como la estadística, computación, psicología cognitiva y la propia bibliotecología y ciencia de la información, entre otras; además de solaparse en su alcance con otras áreas de estudio de la visualización como la de datos, del conocimiento, la científica, etcétera.

Los autores de este estudio igual defienden la idea de que la interdisciplinariedad en la VI se distingue desde distintas perspectivas: aplicada, porque utiliza métodos y teorías de otras disciplinas, y epistemológica porque, aun cuando tiene sus bases en el cognitivismo, permite el análisis desde el sociocognitivismo al visualizar redes sociales, comunidades online, etc., y analizar desde un punto de vista cognitivo la actuación del usuario ante la representación 
de la información en un contexto organizacional o en un dominio específico (Torres, 2008).

El aspecto considerado una actividad relativamente nueva es el uso de las representaciones gráficas, en combinación con las diferentes herramientas informáticas para conseguir visualizaciones de la información más óptimas, lo que constituye uno de los principales objetos de estudio por no pocas disciplinas en los últimos años.

Parte de la credibilidad y sustento de la visualización como disciplina científica se respalda en cinco de sus principios básicos, los cuales dan orden, sentido y comprensión a la VI y a su capacidad para el análisis e interpretación de datos e informaciones. Los cinco principios son el principio de adquisición de conocimiento mediante imágenes, el de fusión, el de transformación de conocimiento, el de objetivización y el del naturalismo, que integrados posibilitan la magia de la visualización y sus múltiples posibilidades.

La VI no es el resultado implícito del acto de ver, no consiste de forma exclusiva en observar. La VI depende mucho del programador y analista de información, el cual debe ser capaz de lograr representaciones gráficas diáfanas y hacer posible la conversión de datos abstractos y fenómenos complejos de la realidad en imágenes visuales comunicativas que permitan ser comprendidas. La VI persigue hacer visible la información casi inapreciable al entendimiento humano, en ello radica su gran esencia y proyección.

Skupin y Fabricant (2003) afirman que la visualización de información es aplicable a una gran variedad de datos, pero la transformación de los mismos hacia una forma visual y la elección de la técnica específica varían en función de las características de los datos (estructurados, semiestructurados o no estructurados). Significa que, para conjuntos de datos con características definidas, se recomienda utilizar en cada caso técnico específicas que se ajusten a las características de los datos.

\section{TÉCNICAS DE VISUALIZACIÓN}

Entre las técnicas de reducción de la dimensión más utilizadas, se encuentran el análisis de cluster, empleado en los mapas de Aaronson; el escalamiento multidimensional (MDS) utilizado por Garfield en sus mapas de la ciencia; el análisis factorial (Kinnucan et al., 1987), utilizado por prestigiosos autores en un estudio publicado en 1987; las redes neuronales como técnica conexionista, también denominada mapas autoorganizativos (som) (Lin et al., 1991).

En los noventa apareció con una mayor fuerza el análisis de redes sociales (White et al., 2000; Chen y Paul, 2001; White, 2003), el cual se fortalece con 
la utilización del algoritmo Pathfinder Networks (Pfnets) para la poda de enlaces en la visualización de redes sociales, obteniendo estructuras similares, pero más informativas (Chen, 1998a; 1998b), etc.; adicionándole también a la técnica de redes sociales la implementación del algoritmo Kamada Kawai para la representación espacial de la información por parte de la comunidad de investigadores.

Todas estas técnicas son complementarias, pues ofrecen diferentes soluciones a un mismo problema (Chen, 1998b: 31). Los estudios que aplican estas técnicas utilizan como unidades de estudio (agregados) autores, revistas, categorías temáticas, palabras claves, resúmenes, co-publicaciones, etc. La capacidad de abstracción a la hora de situar espacialmente los objetos estudiados de acuerdo a las frecuencias de co-aparición de las unidades de estudio es una de las principales características de estos mapas, ofreciéndoles la doble funcionalidad de ser capaces tanto de visualizar la información como de potenciar su recuperación.

En los años noventa, se registró el surgimiento de nuevos métodos y técnicas para la recopilación de la información, análisis, visualización y posicionamiento espacial de la información en el campo de la documentación y ciencia de la información.

Hjorland y Albrechtsen (1995) publicaron en jasis lo que denominaron "nuevo modelo o enfoque para el estudio de la documentación", basado en el estudio de las áreas de conocimiento, desde el punto de vista de la forma de pensar o hablar que tiene la sociedad, posiblemente determinada por su entorno laboral, social, económico, etc., denominándolo análisis de dominios, el cual propone un nuevo paradigma disciplinar que anuncia que la evaluación de la ciencia debe realizarse a partir de las prácticas sociales de los científicos.

El análisis de dominio se basa en el paradigma dominio-analítico que establece, según Hjorland y Albrechtsen (1995), que la mejor forma de comprender la información consiste en estudiar los dominios de conocimiento como parte del discurso de las comunidades de las que proceden, que son el reflejo de la división social y laboral de la sociedad (organización del conocimiento, patrones, estructura, lenguaje, formas de comunicación, entre otras). El paradigma dominio-analítico es, en esencia, social, ya que potencia la perspectiva psicológica, sociolingüística y sociológica de la ciencia; es una aproximación filosófica realista, ya que intenta establecer la base científica de un dominio mediante factores externos a la perspectiva individualista y subjetiva de los usuarios (entra en contradicción con los paradigmas cognitivo y conductivista) (Hjorland y Albrechtsen, 1995). Esta perspectiva se aplicaría a cualquier dominio, independiente de su naturaleza y tamaño, o sea que no 
tiene que estar reducida sólo al área de la documentación, pues aporta una visión holística y objetiva en cualquier dominio que se analice.

Este nuevo frente de investigación está estrechamente relacionado e interconectado con la visualización de dominios, porque esta última aporta las técnicas de apoyo para realizar los análisis de dominio. Y dentro de los once métodos propuestos por Hjorland y Albrechtsen (1995), los estudios bibliométricos son la aproximación más completa para realizar un análisis de un dominio. Hoy hay una fuerte corriente que apoya y fundamenta el enfoque bibliométrico desde la perspectiva del análisis de dominios, junto a la llamada visualización bibliométrica para realizar el análisis y visualización de grandes dominios científicos mediante indicadores bibliométricos (Vargas, 2005).

\section{VISUALIZACIÓN DE MAPAS}

La construcción de mapas a partir de la información bibliométrica se ha llamado cienciografía (scientography), término usado por el responsable de la investigación básica del ISI nombrado George Vladutz, quien lo utilizó para denominar los gráficos o mapas que se obtienen como consecuencia de la combinación de la cienciometría con la geografía (Garfield, 1986).

La cienciometría figura como una especialidad basada en la citación, cuyo objetivo es el estudio cuantitativo de la producción científica mediante la aplicación de técnicas bibliométricas a la literatura científica. A pesar de ser poco utilizado, el término cienciografía, por existir otros más populares, como visualización de dominios o visualización del conocimiento, que conciben y hacen alusión a la misma idea y objetivos, esta investigación considera que dicho vocablo describe muy bien el hecho de cartografiar la ciencia mediante grafos (scientogramas), para que sea visualizada (visualización de dominios científicos) y analizada (análisis de dominio) (Garfield, 1986: 47).

La cienciografía es considerada para el análisis de dominio: herramienta y método. Herramienta porque permite la elaboración y generación de mapas bibliométricos, constituyendo a su vez una herramienta holística, ya que permite construir estos mapas a partir del discurso de la comunidad a representar; y una herramienta objetiva porque esos mapas se construyen a partir de los criterios y opiniones de los sujetos de la comunidad analizada. Método porque auxilia el análisis de dominio al mostrar de forma gráfica la estructura y relaciones de los elementos presentes en el dominio, y considerada método holístico porque permite analizar el dominio a partir del discurso de la comunidad en que se forma, valorada también como método objetivo, pues posibilita el análisis de la estructura no subjetiva por medio 
del consenso intelectual de las relaciones existentes entre los elementos que lo representan.

La simbiosis entre herramienta-metodología y entre holisticismo-objetivismo presentes en la cienciometría, se fortalecen más mediante el uso de redes sociales en la representación gráfica de los mapas bibliométricos (Garfield, 1986: 47). Las redes posibilitan representar los dominios no sólo como grupos de elementos aislados para estudiar exclusivamente sus características, sino que permite, además, estudiar el dominio como conjuntos completos, donde, además de las características, se identifican las relaciones entre sí, llegando a conclusiones cada vez más sustanciosas.

Existen muchas definiciones de este tipo de mapas, pero en esencia todas coinciden en que los mapas bibliométricos son representaciones espaciales nacidas como resultado de la aplicación de indicadores bibliométricos, los cuales permiten llevar la praxis de un dominio x a una representación espacial. Por ello, los mapas no son un objetivos en sí mismos, sino que son el medio mediante el cual se recuperan y representan estructuras del conocimiento, sirve de herramienta para expresar visualmente las relaciones que se establecen entre las diferentes variables objeto de estudio.

Estos mapas bibliométricos ofrecen la posibilidad de hacer representaciones y combinar cualquiera de los elementos que componen los diferentes campos de un registro bibliográfico. Representaciones que son posibles por la co-ocurrencia de determinados elementos en los registros de las bases de datos. Estos mapas se elaboran a partir de la premisa de que los valores de las co-ocurrencias entre dos agregados cualesquiera deben ser considerados como el inverso de la distancia (similaridad) existente entre esos mismos agregados en una representación bidimensional. Esta relación inversamente proporcional permite utilizar diversos procedimientos matemáticos para calcular las coordenadas de los agregados en el mapa, a partir de sus distancias (Chinchilla, 2004). Esto permite, a la vez, mediante la utilización de una metáfora espacial, representar las relaciones existentes entre las unidades objeto de estudio.

Las metáforas visuales dependen de los algoritmos aplicados, de ahí que existan diferentes formas de visualizar datos. Registrados en la literatura siete métodos generales de representación visual: 1-D (unidimensional), 2D (bidimensional), 3-D (tridimensional), multidimensional, árboles, redes y aproximaciones temporales (Shneiderman y Plaisant, 2006). En función del algoritmo aplicado y la metáfora visual utilizada así será la representación espacial que se obtenga.

La mayor parte de las representaciones visuales se enfoca en el análisis de datos multivariables, su clasificación y al reconocimiento de patrones en la 
visualización de dominios científicos, pero la denominación de las interfaces visuales aún tiene imprecisiones, sin distinción se nombran mapas de cocitación, co-ocurrencias, conocimiento, dominios del conocimiento, tecnológicos y demás. En esta investigación se detectó que se orienta más su calificativo al indicador bibliométrico utilizado, que al algoritmo o metáfora visual empleada en la representación espacial.

White y McCain $(1997 ; 1998)$ publicaron dos artículos en los que proponían la visualización como modelo para estudiar y analizar una disciplina mediante mapas temporales. Garfield (1998) aboga por el uso de las nuevas técnicas de visualización para la generación de mapas globales-secuenciales de la ciencia, él plantea que las nuevas técnicas de visualización hacen posible la generación de mapas globales de la ciencia que al hacer zoom sobre ellos o representar distintos periodos, permiten identificar los frentes de investigación emergentes y asociar nombres de autores a cada frente (Garfield, 1998).

Es una realidad que el uso de los mapas como metáforas de una disciplina científica tiene un gran potencial como interfaz para la recuperación de información (Noyons et al., 1999; Buter y Noyons, 2001; Noyons, 2001). Sobre este último aspecto, Chen incorpora el Pfnets al campo de la documentación para la poda de enlaces en la visualización de redes sociales (Chen, 1998a). Más tarde, White propone Pfnet como una nueva técnica para realizar mapas de cocitación de autores (aca) y sus ventajas (Chen (1998a: 41). En ese mismo año, Chen analiza y compara, junto a Morris, el Minimum Spaning Tree (mst) con Pfnets para la detección e identificación de frentes de investigación, concluyendo que el algoritmo Pfnets ofrece resultados más estables (Chen y Morris, 2003), criterio compartido por esta investigación. Noyons (2001) encamina un estudio con otros colegas, en el que analizan el uso de los mapas como metáfora de una disciplina científica, se exponen sus usos como interfase y sus posibles limitaciones (Chen y Morris, 2003: 56, 58).

Entrado el nuevo milenio, comienzan a proliferar estudios basados en la visualización de pequeños dominios científicos. En 2000, White da a conocer las redes centradas en un sujeto, argumenta que los mapas de dominio siempre se habían elaborado a partir de los autores más citados de un grupo seleccionado de publicaciones, proponen entonces como alternativa que se hicieran a partir del nombre de un autor (White, 2000). Un año más tarde, Chen et al. (2001) describen el desarrollo de un método que amplía y transforma el análisis tradicional de coautores en patrones estructurales de la literatura científica, los cuales se representarían en mapas 3D y facilitar la obtención de un mapa del conocimiento claro y más fácil de interpretar (Chen y Paul, 2001). Para estos autores, las nuevas técnicas de visualización permitieron representar mejor la esencia del conocimiento, se perfecciona el 
proceso de modelización y representación de la estructura o mapa intelectual de cualquier dominio, ya fuese geográfico, temático o intelectual.

Otros casos de estudio encontrados en este periodo son, por ejemplo, el trabajo de Ding (et al., 1999), quienes elaboraron un mapa de la estructura intelectual del campo de la recuperación de la información en un periodo de diez años (1987-1997). Ellos muestran modelos, patrones y tendencias del campo analizado, así como distintas medidas del grado de asociación entre los términos más relevantes de los documentos producidos bajo el epígrafe "recuperación de información" (Ding et al., 1999).

Por otra parte, Rerup y Larsen (2001) investigan las ventajas de la visualización gráfica de la producción científica de un área geográfica y utilizan MDS respecto de los métodos tradicionales. En este mismo sentido, otros autores prueban con otra técnica, describiendo un método en el que los documentos de una base de datos sean clasificados automáticamente mediante un som, se subraya la posibilidad de éste para utilizarlo en la clasificación, browsing y recuperación de información en esa base de datos (Guerrero et al., 2002). Continúan el desarrollo y perfeccionamiento de las técnicas White, Buzydlowski y Lin basándose en la experiencia acumulada por White en los trabajos citados y en sus cameos, después implementan un sistema dinámico de visualización: el Authorlink, sistema basado en la co-citación de autores que permite la búsqueda y la recuperación de información en tiempo real (Guerrerero et al., 2002: 39; Buzydlowski et al., 2002).

Un año más tarde, Small (2003) también insiste en las herramientas y, al respecto, teoriza sobre el diseño de una herramienta web capaz de detectar y monitorizar en tiempo real cambios en los frentes de investigación de un área producto en sus interacciones. Otros especialistas reafirman la posibilidad del uso de la citación y la co-citación para estudiar la aparición y evolución de nuevos frentes de investigación a través del tiempo, ejemplificando sus criterios mediante un estudio en el campo de la física (Chen y Kuljis, 2003). Morris et al. (2003), junto a otros investigadores, trabajan con la visualización orientada a la detección e identificación de los cambios temporales en los frentes de investigación.

Börner et al. (2003: 8) revisan magistralmente las diferentes técnicas para el análisis, visualización y posicionamiento espacial de la información. Boyack y Börner (2003) también elaboran, con fines evaluativos, mapas de publicaciones científicas subvencionadas, y logran hacer visible la relación entre el financiamiento gubernamental y el número de citas recibidas. Y dos años más tarde, ambos autores, junto a Boyack et al. (2005), desarrollaron una metodología que permite la visualización a "vuelo de pájaro" del mapa de la ciencia mundial. 
Los mapas, según Börner, se utilizan para identificar objetivamente áreas principales de investigación, expertos, instituciones, colecciones, becas, artículos, revistas, ideas y demás, en un dominio de interés.

Una de las aportaciones más valiosas del grupo de investigación Scimago (De Moya et al., 2004; 2005) fue la propuesta de utilizar la co-citación de categorías ISI como unidades de análisis y representación para la generación de mapas de grandes dominios científicos. Ellos sugieren que las visualizaciones construidas con espacios temporales más cortos eran mejores para el estudio de la evolución de un determinado dominio científico. Criterio respaldado por la teoría que varios mapas de un mismo objeto de estudio son mejores que uno solo, porque así cada mapa proporciona una perspectiva diferente del dominio bajo análisis. Esta línea de investigación es seguida por Leydesdorff (2007) unos años más tarde, quien combina el Journal Citation Report del SCI y del SCI de 2004, y a partir de los mapas de revistas que obtiene muestra sus especialidades de forma muy coherente.

Y, por último, dentro de los diferentes tipos de mapas existentes y que se deben seguir perfeccionando, los más estudiados son los mapas de citas (Leydesdorff, 2007: 9, 11, 12), que intentan identificar factores externos a la percepción subjetiva del usuario, rompen los esquemas mentales apriorísticos para representar una realidad que no se percibe de antemano (Leydesdorff, 2007: 54, 8, 78); mapas de co-ocurrencia de palabras, los cuales se aplican cuando se desea reducir un texto a las palabras o sus relaciones mediante las apariciones conjuntas de las palabras que componen ese texto, se identifican áreas emergentes de investigación, composición de los campos científicos, entre otros (Callon et al., 1983; Braam et al., 1991a; Leydesdorff y Heimeriks, 2001; Ding et al., 2001; Kim et al., 2008); mapas de colaboración, suponen un análisis más sofisticado de los datos y permiten mostrar las redes de colaboración entre las unidades objeto de estudio, la situación central o periférica de cada uno de ellos y la agrupación de agregados con similares patrones de colaboración (Bellavista et al., 1997; Bordons et al., 1996; Glänzel; 2000; 2001); mapas del conocimiento, los cuales analizan en el campo bibliométrico el conocimiento que se encuentra reflejado en las publicaciones científicas y almacenado en las bases de datos o internet, además de los mapas de dominios del conocimiento que también se basan en el análisis de co-ocurrencias de palabras para modelar y representar estructuras intelectuales (Glänzel, 2001: 41, 8, 78); mapas conceptuales, como estructuras que permiten una relación entre conceptos e ideas, orientados a los espacios semánticos. Aquí un concepto cercano a otro indicaría una relación e identificar dentro de un área científica los campos de conceptos más importantes y sus asociaciones (Kopcsa et al., 1999; Ferrer et al., 2005; Tejada et al., 2008); mapas cientifi- 
co-tecnológicos, que representan los input-output de la ciencia a través de indicadores. Esta investigación considera los mapas tecnológicos que proceden de forma exclusiva de la aplicación y de los análisis de indicadores de patentes (Glänzel y Meyer, 2003; Leydesdorff y Meyer, 2007; Díaz, 2007; Díaz y de Moya, 2008; Díaz et al., 2010; Díaz, 2010).

\section{Conclusiones}

Los métodos teóricos aplicados en esta investigación permitieron comprender la diferencia o especialización que se percibe en la visualización bibliométrica, la cual, además de mejorar la recuperación y representación de grandes volúmenes de datos, tal y como lo hace la visualización de información, persigue, además, mejorar la compresión de los datos que subyacen en los conceptos ya establecidos, mediante técnicas como la co-citación, co-ocurrencia de palabras, entre otros.

El análisis realizado identificó que la tendencia actual persigue desarrollar mapas lo más interactivos posibles, los cuales permitan la navegación intuitiva, la filtración rápida de datos relevantes y la recuperación de datos a pedido.

Cada dominio de análisis requiere acercamientos diversos, dependiendo de sus particularidades e intereses, técnicas de representaciones visuales diferentes, conforme se revisó en este estudio un amplio conjunto de herramientas y sistemas para su implementación. Al respecto, aquí se valora que la técnica más apropiada para visualizar y representar las diferentes estructuras presentes en los estudios de dominios tecnológicos son los mapas de análisis de redes sociales (Social Network Analysis), basados en estudios bibliométricos. La representación visual que ofrece un gráfico de redes proporciona a los investigadores y analistas de información un descubrimiento más diáfano de los patrones y estructuras que subyacen en un dominio, específicamente en los dominios tecnológicos en los que la estructura del conocimiento es aún más compleja, porque abarca la amplia clasificación del conocimiento técnico.

Las visualizaciones de dominios tecnológicos mediante mapas tecnológicos facilita los procesos de análisis y representación de datos, potenciando la gestión de la información de patentes y el descubrimiento de conocimiento; los mapas son capaces de revelar todos los comportamientos que subyacen en un dominio. En términos de mapas tecnológicos, la VI supera las posibilidades que ha ofrecido la gestión tradicional de la tecnología, pues antes las técnicas aplicadas no permitían visualizar el entramado de relaciones que se establecen en los procesos de innovación. 
En la actualidad, la innovación como sistema es un proceso social en el que convergen e interactúan diferentes actores y factores de diferente naturaleza en un entorno que propicia esta integración. Es la inclusión de cada una de sus partes y no su simple suma, obteniendo de esta integración sistémica un nuevo estadio de desarrollo en la solución de los problemas de la sociedad.

Toda esta complejidad presente en las innovaciones y generación de tecnologías no es posible representarla con las técnicas que se han usado de forma tradicional, que, en mayor medida, se ocupaban de porcentajes y cifras macroeconómicas. El contexto del presente exige nuevos retos y funciones, de ahí la necesidad de utilizar técnicas capaces de representar los diferentes contextos y relaciones en los que se desarrollan las tecnologías.

Hoy la mejor forma de representar un escenario innovador es mediante la utilización de técnicas de visualización de redes sociales, cuya aplicación en los dominios tecnológicos permiten mostrar relaciones de coautoría, colaboración, transferencia de tecnologías, capacidades tecnológicas, frentes de investigación, consorcios tecnológicos u otros, que existen en los procesos de innovación tecnológica y que es importante su análisis y representación para el gremio tecnólogo e investigador; así como para realizar óptimamente los estudios de monitoreo y vigilancia tecnológica.

La idea de representar mapas científicos y su comunidad en forma de redes no es nuevo, incluso está en las bases de los planteamientos de Garfield y Price, pero algo novedoso sí lo constituye la aplicación de técnicas de redes sociales al análisis de dominios tecnológicos. Visualizar en mapas de redes el dominio tecnológico de países, empresas, tecnólogos, productos, líneas de investigación u otros es algo muy apreciado y notable en la actualidad, ya que agrega un significativo valor a los estudios métricos con patentes.

Las decisiones estratégicas que se tomen mediante el uso e interpretación de mapas tecnológicos que visualicen el comportamiento de grandes volúmenes de datos procedentes de patentes, descarta la utilización de técnicas manuales a la hora de ejecutar proyectos de investigación e innovación. Además de que la aplicación de técnicas de redes sociales en análisis de dominios tecnológicos permite identificar la construcción de procesos de aprendizaje colectivo en la generación de una tecnología, así como identificar el flujo de conocimiento tecnológico, y la transferencia de conocimientos y capacidades tecnológicas en un dominio; algo de especial relevancia para evaluar y documentar la construcción de políticas en ciencia, tecnología e innovación en una organización, país o región. 


\section{Reconocimientos}

Se reconoce el apoyo en el desarrollo de esta investigación del Programa vlir Red Universitaria de Cooperación del Consejo Inter Universitario Flamenco (vlir), específicamente al proyecto internacional "Fortalecimiento del papel de las tecnologías de la información y de la comunicación (tic) en las universidades cubanas para el desarrollo de la sociedad", Cuba-Bélgica.

\section{REFERENCIAS}

Aaronson, Susan Ariel. 1975. "The footness of science”, Mosaic, vol. 6: 22-27.

Archibugi, Daniele. 1992. "Patenting as an indicator of technological innovation: A review", Science and Public Policy, vol. 19, no. 6: 357-358.

Bellavista, Joan, Elena Guardiola, Aída Méndez y María Bordons. 1997. "Evaluación de la investigación”, Cuadernos Metodológicos del CSI (Madrid: Centro de Investigaciones Sociológicas), vol. 23.

Belver, Griffith C., Henry Small, Judith A. Stonehill y Sandra Dey. 1974. "The structure of scientific literature, II: toward a macro and microestructure for science", Science Studies, vol. 4:339-365.

Birger, Hjorland y Hanne Albrechtsen. 1995. "A new horizon in information science: domain analysis", Journal of the American Society for Information Science (JASIS), vol. 46, no. 6: 400-425.

Bordons, María, Isabel Gómez, María Fernández, María Ángeles Zulueta y Aída Méndez. 1996. "Local, domestic and internacional scientific collaboration in biomedical-research", Scientometrics, vol. 37, no. 2: 279-295.

Börner, Katty, Chaomei Chen y Kevin W. Boyack. 2003. "Visualizing knowledge domains”, Annual Review of Information Science and Technology, vol. 37, no.5: 179-255.

Boyack, Kevin W. y Katty Börner. 2003. "Indicator-assisted evalution and funding of research: visualizing the influence of grants on the number and citation counts of research papers", Journal of the American Society for Information Science and Technology (JASIST), vol. 54, no. 5: 447-461.

Boyack, Kevin W., Jochen Kla y Katty Börner. 2005. "Mapping the Backbone of Science", Scientometrics, vol. 64, no.3:351-374.

Braam, Robert R., Henk F. Moed y Anthony F. J. van Raan. 1991. "Mapping of science by combined co-citation and word analysis. I: structural aspects", Journal of the American Society for Information Science (JASIS), vol. 42, vol. 4: 233-251.

Bradford, Samuel C. 1948. Documentation. Londres: Crosby Lockwood and Sons.

Bush, Vanevar. 1945. "As we may think", The Atlantic Monthly, vol. 176: 101-108, en <http://www. theatlantic.Com/doc/194507/bush>, consultada en septiembre de 2007.

Buter, R.K. y Ed Noyons. 2001. "Improving the functionality of interactive bibliometric science maps”, Scientometrics, vol. 51, no.1: 55-67.

Buzydlowski, Jan W., Howard D. White y Xia Lin. 2002. "Term co-occurrence analysis as an interface for digital libraries", Lecture Notes in Computer Science Series, vol. 2539: 133-144. 
Callon, Michel, Jean-Pierre Courtial, Patricia A. Turner y Fernand Baudin. 1983. "From translation to problematic networks an introduction to co-word analysis", Social network information Sur les Sciencie Sociales, vol.22: 191-235.

Chen, Chaomei. 1998a. "Bridging the gap: the use of pathfinder networks in visual navigation”, Journal of Visual Languages and Computing, vol. 9: 267-286.

Chen, Chaomei. 1998b. "Generalised similarity analysis and pathfinder network scaling”, Interacting with Computers, vol. 10: 107-128.

Chen, Chaomei y Jasna Kuljis. 2003. "The rising landscape: a visual exploration of superstring revolutions in physics", Journal of the American Society for Information Science and Technology (JASIST), vol. 54, no.5: 435-446.

Chen, Chaomei y Ray J. Paul. 2001. "Visualizing a knowledge Domains's Intellectual Structure", Computer, vol. 34, no.3: 65-71.

Chen, Chaomei y Steven A. Morris. 2003. "Visualizing evolving networks: Minimum Spanning Trees versus Pathfinder Networks" IEEE Symposium on information visualization.

Chen, Chaomei, Ray J. Paul y Bob O'Keefe. 2001. "Fitting the jigsaw of citation: information visualization in domain análisis", Journal of the American Society for InformationScience and Technology (JASIST), vol. 52, no.4: 315-330.

Chinchilla, Zaida. 2004. "Análisis del dominio científico de español: 1995-2002 (ISI, Web of Science)”. Granada: Universidad, Departamento de Biblioteconomía y Documentación, tesis doctoral.

Costa, Joaquín. 1998. La esquemática: visualizar la información. Barcelona: Paidós (Paidós Estética, 26).

Brooks, Dana H. 1994. "The relationship between science and technology”, Research Policy, vol. 23: 477-486.

Díaz Pérez, Maidelyn. 2010. "Visualización del análisis del dominio tecnológico de Cuba: 1997-2008. Granada: Universidad de Granada, Departamento de Biblioteconomía y Documentación, tesis doctoral.

Díaz Pérez, Maidelyn. 2007. "Producción tecnológica de América Latina con mayor visibilidad internacional: 1996-2007”. Granada: Universidad de Granada, tesina en opción al Diploma de Estudios Avanzados.

Díaz Pérez, Maidelyn y Félix de Moya-Anegón. 2008. "El análisis de patentes como estrategia oportuna para la toma de decisiones innovadoras”, El Profesional de la Información, vol. 17, no. 1.

Díaz Pérez, Maidelyn, Soleidy Rivero y Félix de Moya-Anegón. 2010. "Producción tecnológica latinoamericana con mayor visibilidad internacional: 1996-2007. Un estudio de caso: Brasil", Revista Española de Documentación Cientifica, vol. 33, no. 1:34-62.

Ding, Ying, Gobinda G. Chowdhury y Schubert Foo. 2001. "Bibliometric cartography of information retrieval research by using co-word analysis", Information processing \& management, vol. 37: 801-817.

Ding, Ying, Gobinda G. Chowdhury y Schubert Foo. 1999. "Mapping the intellectual structure of information retrieval studies: an author cocitation analysis, 1987-1997", Journal of Information Science, vol. 25: 67-78.

Doyle, Lauren B. 1961. "Semantic roadmaps for literature searchers", Journal of the Association for Computing Machinery, vol. 8, no. 4:553-578. 
Eick, Stephen G. 2001. "Visualizing on-line activity", Communications of the ACM, vol. 4 no. 8: $45-50$.

Ferrer Troyano, Francisco Javier, Jesús Salvador Aguilar Ruiz, José Cristóbal Riquelme Santos. 2005. "Connecting segments for visual data exploration and interactive mining of decision rules”, Journal of Universal Computer Science, vol.. 11, no. 11: $1835-1848$.

Garfield, Eugene. 1998. "Mapping the world of science (at the 150 Anniversary Meeting of the AAAS" (Filadelfia), en http://www.garfield.library.upenn.edu/papers/ mapsciworld.html>, consultada en marzo de 2003.

Garfield, Eugene. 1988. "The encyclopedic isi-Atlas of Science launches 3 new sections: bochemistry, inmunology, and animal and plant sciences", Current Contents, vol. 7:3-8.

Garfield, Eugene. 1986. "Towards scientography”, Essays of an Information Scientist, vol. 9: 324 .

Garfield, Eugene. 1984. "Introducing the ISI Atlas of Science: Biotechnology and molecular genetics, 1981/82 and bibliographic update for 1983/84", Current Contents, vol. 41:3-15.

Garfield, Eugene. 1981. "Introducing the isi Atlas of Science: Biochemistry and molecular biology 1978-80", Current Contents, vol. 42: 5-13.

Garfield, Eugene. 1963. "Citation indexes in sociological and historical research", American Documentation, vol. 14, no. 4: 289-291.

Garfield, Eugene, Irving H. Sher y Richard J. Torpie. 1964. The use of citation data in writing the history of science. Filadelfia: Institute for Scientific Information.

Gershon, Nahum y Ward Page. 2001. "What storytelling can do for information visualization?", Communications of the ACM, vol. 44, no. 8: 37.

Glänzel, Wolfgang. 2001. "National characteristics in International Scientific Co Authorship Relations”, Scientometrics, vol. 51, no. 1: 69-115.

Glänzel, Wolfgang. 2000. "Science in Scandinavia: A Bibliometric approach”, Scientometrics, vol. 48, no. 2: 121-150.

Glänzel, Wolfgang y Martin Meyer 2003. "Patents cited in the scientific literature: An exploratory study of reverse citation relations", Scientometrics, vol. 58, no. 2: 415-428.

Glenberg, Arthur y William Langston. 1992. "Comprehension of illustrated text: Pictures help to build mental models", Journal of Memory and Language, vol. 31: 129-151.

Griliches, Zvi. 1990. "R\&D, patent statistics as economic indicators”, Journal of Economic Literature, vol. 28, no. 4: 1.661-1.797.

Guerrero-Bote, Vicente, Félix de Moya-Anegón y Víctor Herrero. 2002. “Document organization using Kohonen's algorithm”, Information Processing \& Management, 38: 79-89.

Hearst, Marti A. 2003. "Information visualization: Principles, promise, and pragmatics", Conference on Human Factors in Computing Systems (CHI).

Herrero-Solana, Víctor. 2000. "Modelos de representación visual de la información bibliográfica: aproximaciones multivariante y conexionistas”. Granada: Universidad de Granada, Departamento de Biblioteconomía y Documentación, tesis doctoral. 
Kim, Gil, Jong Hwan Suh y Sang Chan Park. 2008. "Visualization of patent analysis for emerging technology”, Expert Systems with Applications, vol. 34: 1804-181.

Kinnucan, Mark T., Michael J. Nelson y Bryce L. Allen. 1987. "Statistical-Methodos in Information Sciencie", Annual Review and Information sciencie and Technolo$g y$, vol. 22: 147-178.

Kopcsa, Alexander, Edgar Schiebel, Clemens Widhalm y Noll, M.. 1999. "Bibliometric Technology Monitoring", en Workshop by the European Forum for Advanced Business Communications (EEMA): A learning experience centred on Knowledge Management, Múnich.

Larkin, Jill H. y Herbert A. Simon. 1987. "Why a diagram is (sometimes) worth 10,000 words", Cognitive Science, vol. 11: 65-100.

Leydesdorff, Loet. 2007. "Mapping interdisciplinarity at the interfaces between the Science Citation Index and the Social Science Citation Index", Scientometrics, vol.71, no. 3 .

Leydesdorff, Loed y Martin Meyer. 2007. "The scientometrics of a Triple Helix of university-industry-government relations (Introduction to the topical issue)", Scientometrics, vol. 70, no. 2: 207-222.

Leydesdorff, Loed y Gaston Heimeriks. 2001. "The self organization of the European Information Society: The case of 'Biotechnology'", Journal of the American Society for information Science and Technology, vol. 52, no. 14: 1262-1274.

Lin, Xia, Dagobert Soergel y Gary Marchionini. 1991. "A self-organizing semantic map for information retrieval". Chicago: Proceedings of the Fourteenth Annual International ACM/SIGIR Conference Research and Development in Information Retrieval.

Marshakova, Irina. 1973. "System of document connection bassed on references", Nauchno-Teknichescaya Informatisya, series II,no. 6:3-8.

McCormick, Bruce H., Thomas A. DeFanti y Maxine D. Brown. 1987. "Visualization in Scientific Computing", SIGGRAPH Computer Graphic Newsletter, vol. 21, no. 6: $17-32$.

Meyer, Martin. 2006. "Academic Inventiveness and Entrepreneurship: On the Importance of Start-up Companies in Commercializing Academic Patents", Journal of Technology Transfer, vol. 31:501-510.

Meyer, Martin, Tiago Santos Pereirac, Olle Persson y Ove Granstrand. 2004. "The scientometric world of Keith Pavitt A tribute to his contributions to research policy and patent analysis", Research Policy, vol. 33: 1405-1417.

Meyer, Martin y Sujit Bhattacharya. 2006. "Commonalities and differences between scholarly and technical collaboration An exploration of co-invention and co-authorship analyses", Scientometrics, vol. 61, 3: 443-456.

Moreiro, José Antonio. 1998. Introducción al estudio de la información y la documentación. Medellín: Universidad de Antioquia.

Morris, Steven A., G. Yen, Zheng Wu y Benyam Asnake. 2003. "Time line visualization of research fronts", Journal of the American Society for Information Science and Technology (JASIST), vol. 54, no. 5: 413-422.

Moya-Anegón, Félix de, Benjamín Vargas, Zaida Chinchilla, Elena Corera, Francisco Muñoz. 2005. "Domain analysis and information retrieval through the construction of heliocentric maps based on ISI-JCR category cocitation", Information Processing \& Management, vol. 41, no. 6: 1520-1533. 
Moya-Anegón, Félix de, Benjamín Vargas, Zaida Chinchilla, Elena Corera, Francisco Muñoz y Victor Herrero. 2004. "Co-citación de clases y categorías: Proyecto Atlas de la Ciencia”, Scientometrics, vol. 1: 129-145.

Narin, Francis. 1994. "Patent bibliometrics", Scientometrics, vol. 30, no. 1: 147-155.

Narin, Francis, Kimberly S Hamilton y Dominic Olivastro. 1995. "Linkage between agency supported research and patented industrial technology", Research Evaluation, vol. 5: 183-187.

Narin, Francis, Kimberly S. Hamilton y Dominic Olivastro. 1997. "The increasing linkage between U.S. technology and public science”, Research Policy, vol. 26: 317-330.

Noyons, Ed. 2001. "Bibliometric mapping of science in a science policy context", Scientometrics, vol. 50, no. 1: 83-98.

Noyons, Ed, Henk F. Moed y Marc Luwel. 1999. "Combining mapping and citation analysis for evaluative bibliometric purposes: a biliometric study", Journal of the American Society for Information Science (JASIS), vol. 50, no. 2: 115-131.

Polanco, Xavier y A. Zarti. 2008. "Information visualization. State of the art, part C: WP9”. EICSTES Project-IST-1999.

Rerup Ingwersen, Peter Emil y Birger Larsen. 2001. "Mapping national research profiles in social science disciplines", Journal of Documentation, vol. 57, no. 6: 715-740.

Robertson, George G., Stuart K. Card, Jock D. Mackinlay y Ben Shneiderman. 1999. Readings in informatión visualization: using vision to think. San Francisco: Morgan Kaufmann.

Shneiderman, Ben y Catherine Plaisant. 2006. "Strategies for evaluating information visualization tools: multi-dimensional in-depth long-term case studies". Nueva York: Proceedings of the 2006 AVI Workshop on Beyond Time and Errors: Novel Evaluation Methods For information Visualization BELIV '06, ACM Press.

Skupin, André y Sara Fabrikant. 2003. "Spatialization Methods. A Cartographic Research Agenda for Non-geographic Information Visualization”, Cartographic and Geographic Information Science, vol. 30, no. 2: 95-115..

Small, Henry. 1973. "Co-citation in the scientific literature: a new measure of the relationship between two documents", Journal of the American Society for Information Science (JASIS), vol. 24: 265-269.

Small, Henry. 2003. "Paradigms, citations and maps of science: a personal history", Journal of the American Society for Information Science and Technology (JASIST), vol. 54, no. 5: 394-399.

Small, Henry y Belver C. Griffith. 1974. "The structure of scientific literature, I: identifing and graphing specialities", Science Studies, vol. 4: 17-40.

Solla Price, Derek John de. 1965. "Netwoks of scientific papers", Science, vol. 149: 510-515.

Stuart, Toby E. y Joel M. Podolny. 1996. "Local search and the evolution of technological capabilities”, Strateg. Manage. J., vol. 17: 21-38.

Tejada Artigas, Carlos Miguel, Concepción Mendo Carmona, Zape Razquín, Luis Rodríguez e Isabel Villaseñor. 2008. "Los mapas conceptuales como forma de representación de las competencias profesionales en el marco de la formación universitaria”. La Habana: Congreso Internacional de Información INFO. 
Torres, Daniel. 2008. "Bases y desafíos interdisciplinares en la visualización de la información”. La Habana: Congreso Internacional de Información (INFO).

Torres, Daniel. 2007. "La visualización de la información desde los enfoques de la ergonomía cognitiva”. Granada: Universidad de Granada, tesina en opción al Diploma de Estudios Avanzados.

Trajtenberg, Manuel. 1990. "A penny for your quotes: Patent citations and the value of innovations", RAND. Journal of Economics, vol. 21, no. 1: 172-187.

Vargas, Benjamín. 2005. "Visualización y análisis de grandes dominios científicos mediante redes Pathfinder". Granada: Universidad de Granada, Departamento de Biblioteconomía y Documentación, tesis doctoral.

White, Howard D. 2000. "Toward ego-centered citation analysis", en Blaise Cronin y Helen Borsky Atkins, eds., The Web of Knowledge: A Festschrift in Honor of Eugene Garfield. Medford, N.J.: Information Today, 2000, 475-496.

White, Howard D y Katherine W. McCain. 1997. "Visualization of literatures", Annual Review of Information Systems and Technology (ARIST), vol. 32: 99-168.

White, Howard D y Katherine W. McCain. 1998. "Visualizing a discipline: an author co-citation analysis of information science, 1972-1995", Journal of the American Society for Information Science (JASIS), vol. 49, no. 4:327-355.

White, Howard D. 2003. "Pathfinder Networks and Authors Cocitation Analysis: A Remapping of Paradigmatic Information Scientisf", Journal of the American Society for Information Sciencie and Technology (JASIST), vol. 54, no. 5: 423-434.

White, Howard D., Jan Buzydlowski y Xia Lin. 2000. "Co-cited author maps as interfaces to digital libraries: designing Pathfinder Networks in the humanities", ieee International Conference on information visualization.

Zhu, Bin y Chen Hsinchun. 2005. Information Visualization. Annual Review of Information Science and Technology (ARIST), 39: 139-177.

\section{Para citar este texto:}

Díaz Pérez; Maidelyn, Moya Anegón, Félix de; Carrillo-Calvet, Humberto Andrés. 2017. "Técnicas para la visualización de dominios científicos y tecnológicos". Investigación Bibliotecológica: Archivonomía, Bibliotecología e Información (Número Especial de Bibliometría): $17-42$.

http://dx.doi.org/10.22201/iibi.24488321xe.2017.nesp1.57884 